\title{
Metabolic syndrome and perceived quality of life: a case-control study in Ghana
}

\section{Abstract}

Metabolic syndrome (MetS), which refers to co-occurrence of cardiovascular risks primarily obesity, hypertension, insulin resistance and atherogenic dyslipidemia was previously uncommon in sub-Sahara African. But it is increasingly becoming a public health concern heightened by the epidemiological transition associated with excess energy intake, sedentary lifestyles and urbanization. The aim of this study was to assess the dietary, lifestyle and physiologic risk factors associated with MetS and the effect on perceived quality of life. The design was one-to-one age and sex-matched case-control study. MetS was defined as concomitant adiposity, hypertension and diabetes. Cases $(n=76)$ were recruited in the diabetic clinic of a municipal hospital in Ghana while controls $(n=76)$ were recruited in the communities in which the cases lived. Lifestyle habits and physical activity were assessed using a validated lifestyle and habits questionnaire while habitual dietary intakes were assessed using a food frequency questionnaire. Anthropometry, blood pressure and fasting capillary glucose were measured. Differences between cases and controls were tested using McNemar and paired t-test tests. Crude odds ratio (COR) for MetS was estimated using conditional logistic regression.

Mean age of participants was $57.31 \pm 5.46$ years (range $45-65$ years). Females constituted the majority $(95 \%, n=144)$. Among the cases and controls, lateral obesity ( $100 \%$ vs $60.5 \%)$, central obesity $(98.7 \%$ vs $36.8 \%)$, hypertension $(90.8 \%$ vs $2.6 \%)$ and diabetes $(72.4 \%$ vs $1.3 \%$ ) differed significantly. Fewer cases $(10.5 \%)$ consumed diversified and healthy diets compared to the controls $(77.6 \%)$ $(p=0.001)$. Significant number of cases felt a high risk for illness based on how they assessed their physical health $(73.7 \%$ vs $2.6 \%)$, exercise and fitness regime $(88.2 \%$ vs $13.2 \%)$, nutrition and weight control (14.5\% vs $1.3 \%)$ and psychological health $(19.7 \%$ vs $0 \%)$. However, none felt socially unhealthy. Lower risk for MetS was found among participants who had at least secondary education (COR:0.17 95\% CI:0.04-0.73) and lived in a peri-urban setting (COR:0.22 95\% CI:0.09-0.53) while unemployment (COR:9.00 95\% CI:1.26-64.35) and central obesity (COR: 48.0 95\% CI: 6.62-147.74) were associated with higher risk. MetS adversely affected general sense of wellbeing of the middle age adults. Obesity was an important predictor. Educational interventions to promote diversification of dietary intakes and enhance weight control are crucial to reduce MetS and could improve the quality of life of those affected. These interventions should be integrated into already existing community-based social support, and health systems.

\section{Conflict of Interest}

There is no conflict of interest. 